\title{
Dynamic Impact Testing of Hedgehog Spines Using a Dual-Arm Crash Pendulum
}

\author{
Nathan B. Swift ${ }^{a}$, Bor-Kai Hsiung ${ }^{b}$, Emily B. Kennedy ${ }^{b}$, Kwek-Tze Tan ${ }^{c^{*}}$ \\ a Department of Physics, Science Technology Entrepreneurship Master's Program, Case Western Reserve University, \\ Cleveland, OH 44106, USA \\ ${ }^{b}$ Department of Biology, Integrated Bioscience PhD Program, The University of Akron, Akron, OH 44325, USA \\ ' Department of M echanical Engineering, The University of Akron, Akron, OH 44325, USA \\ *Corresponding author: Tel: + 1-330-972-7184; Fax: +11-330-972-6027; E-mail address: ktan@uakron.edu
}

\begin{abstract}
Hedgehog spines are a potential model for impact resistant structures and material. While previous studies have examined static mechanical properties of individual spines, actual collision tests on spines analogous to those observed in the wild have not previously beeninvestigated. In this study, samples of roughly 130 keratin spines were mounted vertically in thin substrates to mimic the natural spine layout on hedgehogs. A weighted crash pendulum wasemployed to induce and measure the effects of repeated collisions against samples, with the aim to evaluate the influence of various parameters including humidity effect, impact energy, and substrate hardness. Results reveal that softer samples-due to humidity conditioning and/or substrate material used-exhibit greater durability over multiple impacts, while the more rigid samples exhibit greater energy absorption performance at the expense of durability. This trend is exaggerated during high-energy collisions. Comparison of the results to baseline tests with industry standard impact absorbing foam, wherein the spines exhibitsimilar energy absorption, verifies the dynamic impact absorption capabilities of hedgehog spines and their candidacy as a structural model for engineered impact technology.
\end{abstract}

Keywords: Impact pendulum; Hedgehog spines; Energy absorption; Biomimicry; Keratin; Humidity

\section{Introduction}

Hedgehog spinesare naturally impact resistant. In the wild, hedgehogs climb trees and plants in search of food (Matthews 1963; Vincent and Owers 1986). They often fall (or jump to avoid predators) from heights exceeding ten meters. A falling hedgehog rolls into a ball and uses its dorsal muscles to erect its spines before impacting the ground at speeds up to $15 \mathrm{~m} / \mathrm{s}$ (Matthews 1963, 1974; Vincent and Owers 1986).Despite the velocity at impact, the animal survives unscathed due to the shock-absorbing capabilities of its spines, which buckle under load (Vincent and Owers 1986; Karam and Gibson 1994).Clearly, these spines serve a vital 
purpose beyond their ability to stab predators with their tapered ends (Matthews 1963), especially considering how difficult it is to remove a hedgehog spine (Carlier 1893) versus a porcupine quill, which functions solely as a weapon (Cho et al. 2012).Made of alpha-keratin, hedgehog spines have a unique internal morphology (Figure 1) (Gibson, Ashby, and Harley 2010). When force is applied axially to an individual spine, it begins to "bow" laterally-closely following the Euler buckling model-once the critical buckling load is achieved. If greater force is applied, the spine will continue to bow until roughly 200 times the critical buckling load is applied, at which point the spine ovalizes (characterized by the Brazier effect) and fails, buckling locally.In testing the longitudinal strength of spines with internal septa removed, Vincent and Owers (1986) discovered that spines failedunder far less axial load than with septa present. They concluded thatit is due to the internal morphology that failure is delayed to such a great magnitudebeyond the critical buckling load, as circumferential septa resist tensile load and reinforce the spine's cylindrical shape (Brazier 1927; Calladine 1983; Vincent and Owers 1986; Ashby 2005; Gibson, Ashby, and Harley 2010).

Measuring $1 \mathrm{~mm}$ diameter, $15-20 \mathrm{~mm}$ length(Vincent and Owers 1986) and weighing an average $3.5 \mathrm{mg}$, a hedgehog spine is a potential model for innovation of ahigh strength-to-weight ratio, impact resistantstructures. It is likely that engineered structures based on hedgehog spines would be more mechanically-efficient in terms of specific strength(Karam and Gibson 1994; Gibson, Ashby, and Harley 2010), material-efficient, and lighter weightthan conventional structures (Beukers and van Hinte 2005). Impact related studies in both engineered and natural armor materials have attracted great attention in recent time (Chen et al. 2011; Song, Ortiz and Boyce 2011; Huang, Durden and Chowdhdury 2011; Chintapalli et al. 2014; Rudykh, Ortiz and Boyce 2015; Bruet et al. 2008).

Few experimental studies have been conducted on the mechanical properties of hedgehog spines. Vincent and Owers (1986), and Karam and Gibson (1994) conducted compression and bending tests on hedgehog spines, finding that the critical Euler buckling force for a single spine is roughly $6 \mathrm{~N}$ and that it takes 200 times the critical buckling load to initiate the Brazier effect(Vincent and Owers 1986).Aside from additional verification by the aforementioned researchers, as well as some similar studies on the static mechanical properties of porcupine quills (Chou and Overfelt 2011; Chou, Overfelt, and Miller 2012; Yang, Chao and McKittrick 2013; Torres et al. 2014), there areno other documented mechanical tests on hedgehog spines and no literature on dynamic behavior of hedgehog spines during impact. However, understanding hedgehog spines' dynamic properties is of utmost importance for assessing their impact protection capabilities, which depend largely on an object's acceleration and mechanical energy absorption(Guskiewiczand Mihalik 2011). Durability-consistent performance across multiple impacts-is also important for many applications such as concussion mitigation in football helmets (Pellman et al. 2004). However, Hedgehog spine durability has not been investigated. Furthermore, Vincent andOwers (1986) and Karam andGibson(1994) only tested static properties of individual spines instead of many spines grouped together similar to the arrangement on a hedgehog pelt(Figure 2), which typically have thousands of densely packed 
spines. Yet, there is reason to believe from observational evidence that spines'systematic grouping enhances their impact protection capabilities.Also, it is known that keratin's mechanical properties are affected by relative humidity of the material, wherein increased humidity generally softens keratin, while decreased humidity makes keratin harder and more brittle (Curiskis and Feughelman 1983;M cKittrick et al. 2012). In similar context, porcupine quills, which are also made of keratin and consist of a hard outer shell (cortex) and compliant porous core (medulla), exhibit considerably lower modulus and strength with increased humidity under axial loading (Chou and Overfelt 2011; Chou, Overfelt, and Miller 2012; Torres et al. 2014).Humidity's effect on strength and energy absorption, durability, and failure pointof hedgehog spines, however, has not been investigated. Adding to that, impact performance of hedgehog spines has not been compared to standard impact protection materials.

In this paper, a dual-arm "crash pendulum" is employed to measure the amount of energy absorbed by spine arrays embedded in a substrate across a variety of parameters, including substrate hardness, kinetic impact energy, relative humidity of the spines, and the effect of multiple hits. This work aims to fill the gaps in knowledge about hedgehog spines' dynamic mechanics. The investigation involving a harder substrate examines how spines alone absorb impact energy by breaking since the epoxy provides fixed and rigid support boundary condition. The softer PU substrate, however, provides a simply support boundary condition, and works more as a system with the spines, contributing to impact absorption when allowing bending at the base of the spines, which more closely resembles spine-in-pelt behavior.

This work aims to provide scholarly contributions in the following areas:

1. First experimental demonstration to show that hedgehog spines function as an impact energy absorber.

2. First study of hedgehog spines as a group system, rather than individual spines, like in previous studies.

3. Identify key coupling elements and parameters to improve and influence the impact absorption performance of hedgehog spines system.

4. Intimate emphasis on Biomimicry and reveal how engineered structural materials can benefit through Biomimicry approach.

\section{Methodology}

\subsection{Sample Preparation}

Intact spines were collected from anaturally deceasedthree-year old female African Pygmy hedgehog (Atelerixalbiventris/algirus) donated to the study from Lawrence, KS.The pelt with all spines attached was skinned from the animal immediately following its death and stored in an oven set at $60^{\circ} \mathrm{C}$ for two weeks to facilitate plucking(Carlier 1893; M atthews 1963).Spines were individually plucked as needed for each sample, with each spine averaging about $15 \mathrm{~mm}$ in length.Spines sampled from all regions of the pelt exhibited similar length and diameter 
(I=15.70 $\pm 0.63 \mathrm{~mm}, \mathrm{~d}=0.98 \pm 0.06 \mathrm{~mm}, \mathrm{~N}=53)$ so region of origin played no part in spine selection. Geographical uniformity of spines is corroborated by Vincent \& Owers (1986).Spines could not be left in the pelt for impact testing due to the variable size, thickness and brittleness of the pelt as it decomposed, making it difficult to mount to the apparatus and adversely affecting data reliability. Using molded substrates, on the other hand, provided a consistent, flat, and sturdy base with unvarying mechanical properties.Test samples were generated by first mounting 130 spines vertically with the sharp end into a piece of packing foam.Next, the foam with spines was flipped and placed over a $5 \mathrm{~cm}$ diameter circular substrate mold so that the bulbed ends of the spines were held vertically embedded in the 3-5mm thick substrate as it cured for 24 hours(see schematic of process in Figure 3(a), S1). The $5 \mathrm{~cm}$ diameter substrates had sufficient surface area to yield statistically greater energy absorption than the no material baseline.Mounting 130 spines in the $5 \mathrm{~cm}$ diameter substrates approximately mimicked the average spine density on the natural model: 6.6 spines per square centimeter. Based on Vincent and Owers (1986) as well as the authors' own measurements, spine density ranges from 3.911.6 spines per square centimeter, depending on how tightly the animal is rolled into a ball. Two different substrate materials were used: M etLab \#M 135 (M etLab Corp, NY, USA), a hard epoxy rated Shore $82 \mathrm{D}$ provides a fixed and rigid boundary condition to largely isolate the spines' absorption capabilities from that of the substrate, and Forsch URS5180 (Forsch Polymer Corp, CO, USA), a softer, more pliable Shore 80A Polyurethane (PU) that provides a simply supported boundary condition by allowing bending moment at the spine/substrate interface. The softer substrate more closely mimics hedgehog flesh and may contribute to the impact absorption. For each sample, the spines were mounted with the bulb end down into the substrate, again mimicking the natural model. A typical finished sample is shown in Figure 3(b).

To test humidity parameters, some samples were placed in a $100 \%$ relative humidity chamber for 48 hours after the substratewas cured. These conditioned samples had estimated $85-90 \%$ relative humidity during the tests (samples were taken out of the chamber and mounted on the pendulum immediately before each test. A typical test with up to ten repeated impacts was conducted and finished within a 15 minute period), while the unconditioned samples had48$51 \%$ relative humidityaccording to local weather data. ${ }^{1}$ Despite a single hedgehog having thousands of spines, only six samples were generated. A large fraction of spines broke during extraction and we required intact spines for sample preparation. Also, we selected spines to eliminate variation in spine length and diameter (accepted range for $I=15.5-16.0 \mathrm{~mm}, \mathrm{~d}=0.96$ $1.00 \mathrm{~mm})$.

For a baseline comparison, a layerof foam from a Riddell Speed Classic football helmet was prepared for testing (hereafter referred to as 'Foam'). It was cut down to match the footprint and thickness of the spine samples.

\footnotetext{
${ }^{1}$ The Weather Channel. 2015. "Weather History for KAKR: M onday, July 20, 2015." Weather Underground.http://www.wunderground.com/history/airport/KAKR/2015/7/20/DailyHistory.html?req_city=Akron \&req_state $=0$ H\&req_statename $=\&$ reqdb.zip $=44301 \&$ reqdb. magic $=1 \&$ reqdb.w mo $=99999$
} 


\subsection{Testing Apparatus}

Impact absorption testing on spines was conducted using a two-arm "crash pendulum" (Figure 4) similar to the setup in Ucar and Cengiz (2012). The system was designed to produce an axially impact loading without gravity effect during impact (Ucar and Cengiz 2012).The crash pendulum can produce impact loading throughout the collision at adjustable velocity and therefore kinetic energy by adjusting the starting height, $h(h=0$ when the pendulum arm hangs straight down at rest). The two pendulum arms with weights bolted to the ends each swing with a radius of $l=3.26 \mathrm{~m}$, connected to hinges by two aluminum extensions in a "V" shape. The total mass of each pendulum arm ism $=43.32 \mathrm{~kg}$. Just above the two weights at roughly the center of mass was a rigid1018 steel sample-mounting surface facing inward toward the other arm. The flat mounting pieces on the two arms were aligned to touch when the pendulum was at rest, hanging vertically under their hinges. Samples were mounted to one pendulum using doublesidedtape. Additionally, a Canon PowerShot SD4500 IS high-speed video camera was mounted on a tripod facing the two pendulum arms, and a backboard with calibrated markings was placed behind the arms to record data throughout each collision.

\subsection{Pendulum Impact Experimental Technique}

Mechanical symmetry between the right and left pendulum arms of the apparatus was established by experimentally testing collisions in both directions.All following trials on the pendulum were conducted by raising the left pendulum and dropping it toward the right pendulum(Figure $4(a)$ ), which initially sat at rest with the sampleattached to its collision plate (Figure 4(b)). All trials were conducted with a left arm starting height $(h)$ at one of two points: 4 degrees for 3 Joule collisions (low energy) and 6.5 degrees for 8 Joule collisions (high energy) (Figure 4(a)-I), then analyzed using two independent techniques.

Using the high-speed camera and calibrated backboard, stopping points for the two pendulum arms were measured post-collision (hereafter referred to as 'potential energy difference experimental technique') (Figure 4(a)-III).Using the assumption that total kinetic energy of the pendulum arms immediately before and after the collision equals the starting and final potential energies, respectively of the arms, the total potential energy was calculated from the arms' starting and stopping angles, where for any given pendulum angle:

$$
U=m g l(1-\cos \theta)
$$

where $U$ is the total potential energy in Joules of the given pendulum arm at its starting or stopping point; $m$ is the total mass in kilograms of the pendulum arm; $g$ is the acceleration due to gravity; $l$ is the length in meters from the pendulum's hinge to its center of mass; and $\theta$ is the pendulum arm's angle in degrees from vertical rest. The small mass addition of the sample mounted to the right pendulum was considered negligible compared to the much larger total mass of the pendulum arm. 
The total potential energy $(U)$ of the arms at the stopping points was compared to the initial potential energy of the left arm at the starting point to determine the energy absorbed $(\in)$ by the material during the collision:

$$
\epsilon=U_{L i}-U_{L f}-U_{R f}
$$

and normalized absorbed energy $\left(\epsilon_{\text {norm }}\right)$,

$$
\epsilon_{\text {norm }}=\frac{\epsilon}{U_{L i}}
$$

where $U_{L i}$ is the starting potential energy of the left pendulum, $U_{L f}$ is the final potential energy of the left pendulum after collision, and $U_{R f}$ is the final potential energy of the right pendulum after collision (Ucar and Cengiz 2012). It is assumed that due to the low velocity of the pendulum arms, air resistance can be ignored (Mohazzabi 2011) and all starting potential energy of the left arm was converted into kinetic energy immediately before collision. Likewise, it is assumedthat all kinetic energy of the two arms immediately after collision was converted back into final potential energy of the two arms when they came to a stop. Other uncertainties that cannot be easily measured, such as the effect of arm vibration or friction in the hinges are accounted for by comparing experimental results to 'Foam,' as well as placing no samples between the two crash pendulum arms sothat only the apparatus itself absorbs energy(hereafter referred to as 'No M aterial'), which will be discussed in section 3.4.

Collisions were repeated on a given sample up to ten times or until the sample was destroyed beyond functionality, as determined through visual assessment (Table 1). In addition, collisions were repeated on the two baselines: 'Foam'and 'No Material', for a performance comparison that accounted for vibrational/frictional uncertainties in the apparatus, as well as to confirm the accuracy of the apparatus by calculating standard error across all hits, which was found to be under $3 \%$.

The pendulum arms' starting and stopping angles in bothdirectionsfrom vertical 'rest' position were recorded manually at the time of experiment and then verified using the high-speed camera footage. These data points were then converted to potential energy UusingEquation 1. Finally, the total energy absorbed $(\epsilon)$ and normalized absorbedenergy $\left(\epsilon_{\text {norm }}\right)$ were plotted against the baselines over consecutive hits of the same sample in order to evaluate sample durability. The research team recorded visual post-mortem observations of sample damage.

The velocity and acceleration of the two pendulum arms throughout the collision were found by analyzing the high-speed camera footage with ProAnalyst software and applying fundamental equations of motion for calculation (hereafter referred to as 'kinetic energy difference experimental technique'). High-speed video footage was recorded by a Canon PowerShot SD4500 IS at the framerate of $240 \mathrm{fps}$ during each pendulum impact trial. Color stickers were applied to the pendulums around the sample-mounting locus for motion tracking. The stickers mirrored each other in terms of their locations on the left and right pendulums (Figure 4(c)). 
ProAnalyst M otion Analysis Software (Xcitex Inc., Cambridge, M A, USA) was used to track each pendulum's stickers frame-by-frame in the high-speed video footage. Coordinate origin was set to the lower left corner of each video recording. Spatial distance in each video was calibrated using a known distance betweenbackboard markings. Pendulum position coordinates $(x, y)$ were exported after each video analysis. Distance travelled for each pendulum between frames (1/240 sec) was calculated using $\sqrt{\Delta x^{2}+\Delta y^{2}}$, and the speed was then calculated accordingly.Speed wasplotted as a function of time for each pendulum (Figure S2) and the resulting curves were smoothed. Acceleration-time curves were generated (Figure S3) by taking first derivativeof the speed-time curves (Figure S2) in GraphPad Prism statistical software (GraphPad Software, Inc., La Jolla, CA, USA). The calculation of kinetic energy different experimental technique is explained in Figure S4. A summary of energy absorption and acceleration values obtained from high-speed video analysis is presented in Table S1.

\section{Results and Discussion}

In this section, the impact test results, as performed according to Table 1,are discussed in terms of the following parameters:

1. Humidity compares performance of the "dry" spines at $48-51 \%$ relative humidity (room environment) versus "humid" spines at $85-90 \%$ humidity.

2. Impact energy compares performance of spines at 3 Joules versus 8 Joules kinetic energy of the left pendulum immediately before impact.

3. Substrate compares performance of spines embedded in the Shore 82D epoxy versus those embedded in Shore 80APUsubstrates.

4. Baseline comparison compares spine samples' performance to 'Foam' and 'No Material'.

\subsection{Humidity}

Two samples of 130 spines each embedded in Shore 82D epoxy are tested for this comparison. The hardness of the epoxy substrate almost entirelyisolates the spines' movement and function, since a fixed and rigid boundary condition is applied.Sample Ahas spines with approximately 48$51 \%$ relative humidity due to storage in normal room conditions, while sample Bcontains spines at $85-90 \%$ relative humidity after being stored in a $100 \%$ humidity chamber for 48 hours prior to the experiment. Each is subjected to five consecutive collisions by the crash pendulum, with impactkinetic energy of 3 Joules.

The impact test results are analyzed independently by two different methods as described in section 2.3 (potential energy difference and kinetic energy difference). Each method has pros and cons: While calculating the kinetic energy difference is the most quantifiable way to analyze the results objectively, and many useful parameters can be extrapolated from the analysis (i.e. 
speed, acceleration, etc.), it does not account for the residual kinetic energy (velocity $\mathrm{V}_{\mathrm{Lf}}$ ) of the left pendulum after collision. On the other hand, estimating energy absorptionusing the potential energy difference experimental techniqueaccounts for $V_{L}$ post-collision, but relies on a visual assessmentof pendulum swing angle based on the backboard reference to determine $h$, and any angle $\theta$ less than 0.5 degree cannot be discerned by this method.The energy absorption results based on both methods, which provide a fuller picture than either method alone,are shown in Figure 5.

Results from both methods showed good agreement in trends of energy absorption over multiple impacts - the energy absorption performance of sample $A$ (dry) constantly decreases over the course of multiple impacts, while that of sample $B$ (humid) remains relatively consistent. It is noted that most spines in sample Abroke and shattered during the second collision, whereas thosein sample Bresumed their original formafter collision. The brittleness of sample Ais attributed to the properties of keratin, wherein the material hardens and stiffens when dried out, and softens and becomes more pliable when wet (Curiskis and Feughelman 1983).

In the third through fifth collisions, theenergy absorption of sample Ais less thanduring initial impact and continuallydegrades, likely due to the fact that most of the spines having already been destroyed by the second collision (Figure6(a)).In contrast, sample Bappears to maintain relatively consistent absorption capabilities throughout the five-collision trial (Figure6(b)). The softer humid spines appear to be more resilient over repeated low-impact energy collisions, though they are unable to absorb as much kinetic energy during the initial collision compared to dry spines. In general, it can be concluded that stiffer spines may offer greater impact energy absorption capabilities, but at the cost of multi-hit durability. The overall estimated normalized absorbed energy $\left(\epsilon_{\text {norm }}\right)$, is higher when calculated from the kinetic energy difference (Figure $5(b))$ because it does not account for post-collision residual velocityv $V_{L}$.

\subsection{Impact Energy}

Next, the influence of different impact speedsis evaluated by introducingtwo new samples of spines-sample C(dry) and D (humid), both prepared in epoxy substrates. In addition, sincethe spines in sample B (humid)remained intact after the lowenergy collisions (Figure $6(b)$ ), they were further investigated to determine their durability by subjecting them to higher energy impacts for additional data points. Again, each sample was subjected to five repeated collisions, each with 8 Joules kinetic impact energy. Sample $C$ was destroyed after two collisions so its use was discontinued.The energy absorption results are presented in Figure 7.

During the first collision,sampleC absorbed a greater amount of energy than either humid spine sample ( $B$ and $D$ ). However,sample Cwas destroyed after only two hits, much more rapidly than both samples with humid spines(Figure 8(a)). This indicates that the greater impact absorption of the dry spines was due to permanent damage. The two humid spine samples ( $B$ and $D$ ), on 
the other hand, did not shatter and resumed their original form after each collision (Figure $6(b)$, $8(b)$ ), demonstrating the resiliency of the softer, humid spines. This is especially so for sample $B$, which endured a total of ten collisions.It is worth noting, however, that unlike inthe low energy test (Figure 5), the humid spines in the high-energy test underwent gradual energy absorption depletion over consecutive hits (Figure 7). Additionally, while all samples absorbed more total energy than during the low energy test, they absorbed a smaller percentage of the initial impact energy (normalized absorbed energy $\left(\epsilon_{\text {norm }}\right)$ during the high-energy test versus the low energy test. This suggests that the capability of hedgehog spines toabsorb energy becomes saturated during high-energycollisions, resulting in greater damage over multiple hits at higher impact speeds.

\subsection{Substrate}

In order to more accurately model the hedgehog impact absorption capabilities, spine samples $\mathrm{E}$ (dry)and $\mathrm{F}$ (humid)were embedded in a softer, more pliable Shore 80A PU substrate.Like the pelt on a hedgehog, the softer substrate may contribute to the impact absorption capabilities of the spine systemby increasing the spines' freedom to rotate at the junctions of the substrate and spine basesand allowing load transfer into the substrate via the embeddedbulbed ends of the hedgehog spines (Vincent and Owers 1986). The two samples were first subjected to five consecutive collisions in low energy tests, and subsequently to five consecutive collisions in high energy tests, since they were still intact (sample $F$ showed no signs of damage while sample $E$ had very minimal damage).

At first glance, the energy absorption results analyzed by two independent methods do not seem to agree with each other in low energy impact tests. The calculated result usingthe kinetic energy difference experimental technique (Figure 9(b)) shows that the energy absorption performance for both sample $E$ and $F$ is relatively consistent throughout five collisions during the test. In contrast, the result calculated using the potential energy difference experimental technique (Figure 9(a)) shows a drastic dip at the third and fourth collision before starting to recover. Recall that the difference between these two methods is the inclusion ofthe left pendulum's residual energy when calculations are performed using the kinetic energy difference experimental technique. By combining results from both methods, the data suggests thatthe energy transferred from the left pendulum to the right across five different collisions remained consistent during the low energy test (Figure 9(b)). The left pendulum's residual energy after each collision steadily increases and peaks at the third and fourth impact before returning to the initial state (Figure 9(a)).The increase of the left pendulum's residual energy can be explained by the softer substrate. In the samples with a softer substrate, spines have more freedom to rotate at the junctions of the substrate and spine bases. Therefore, the early collisions cause the spines to "flatten," or get pushed and reorientedcloser to parallel to the substrate. It is suspected that the movement of the spines limits the amount of energy that can be transferred to the right pendulum during the short collisions, and allows more energy to be 
kept on the left pendulum at the same time. This agrees with our observation from the samples after the test: while few spines - especially those in the humid samples - broke or shattered, it was noted that many spines were flattened (Figure 10), something that did not occur with samples in epoxy substrates. A similar trend was observed in the high energy test (Figure 9(c)), though the change in absorption across collisions is less dramatic.This indicates that the early high energy collisions "flattened" the spines further even when the sample had already endured five collisions in the low energy test. The dip likely signifies the point where the spines have the highest freedom to move before reaching maximum "flatness" .

Evidently, despite the researchers' effort to closely mimic the spine-pelt system of the hedgehog, the PU substrate samples still lacked a control mechanism to keep spines erect from the surface during repeated hits, analogous to the animal's dorsal muscles. Indeed, the rigid epoxy substrate can adequately keep spines erect after impact, as it allows virtually no spine movement. The results suggest that the first hit reoriented the spines embedded in PU because of the greater "wiggle room" in the elastic substrate, and for each hit following, the spines were pushed closer to parallel to the substrate. On a hedgehog, this couldalso happen, except the animal has muscles attached to the base of the spines which it contractsto erect the spines hit after hit(Reeve 1994). As an elastomer, the PU substrate comes closer than epoxy to mimicking the hedgehog pelt system by allowing load transfer into the substrate via the embedded bulbed ends of the hedgehog spines, similar to how impact loads arelikely transferred into the hedgehog skin.

\subsection{Baseline Comparison}

In each of the above experiments, energy absorption results of the samples are plotted against those of a sample of foamfrom a Riddell Speed Classic football helmet cut to match the footprint and thickness of the spine samples ('Foam'), and a second baselinewhere nothing was placed between the two crash pendulum arms and only the apparatus itself absorbed energy ('No Material'). The purpose is two-fold: the 'Foam' baseline helps measure how hedgehog spines perform as an impact material versus industry standard impact absorbing foam, and 'No $M$ aterial' demonstrates the amount of energy absorbed by the apparatus alone due to friction, vibration, or any other unaccounted-for uncertainty, thus giving to the data perspective as the apparatus' "zero point." Despite inherent experimental uncertainties, the results from repeated collisions with 'Foam' and with 'No Material'show consistency throughout the experiment, confirming the repeatability of the pendulum technique. A t-test analysis of the energy absorption between 'Foam' and 'No M aterial' shows that the crash pendulum apparatus has the resolution to tell them apart and further supportstheresults(Figure 11). Spine samples generally performed similarly to'Foam'; indeed some absorbed slightly less while others absorbed slightly more. This confirms hedgehog spines' inherent dynamic impact absorption capabilities. 


\subsection{Protection vs. Durability}

In the human brain, it is known that certain acceleration of the head is likely to cause injury such as concussion (Ommaya and Gennarelli 1974). Therefore, an impact material that minimizes the acceleration experienced during a collision by absorbing as much kinetic energy as possible can reduce the likelihood of concussion, or other similar injury and damage. Figure 12compares the acceleration of the pendulum during impact for each sampleacross all collisions.Overall, during low energy collisions (Figure 12(a)), all samples except the dry epoxy sample experienced significantly lower acceleration when compared to 'No Material.'It seems that at slower collision speeds, the individual parameters tested above on the spine samples make little difference to acceleration.Interestingly, for the high-energy collisions (Figure 12(b)), the exact opposite pattern occurred - only the dry epoxy sample experienced significantly lower acceleration when compared to 'No Material.' One explanation for the seemingly impressive performance of the dry epoxy is that the sample only lasted for two collisions before complete destruction, during which it absorbed more energy than any other sample. The most significant takeaway is that on average, the spines absorb about the same energy as 'Foam,' and therefore reduce acceleration throughout the collision by about as much as 'Foam.'

The other important metric of this study is multi-hit durability. That is, the number of hits that a given sample can endure while maintaining consistent impact absorption performance. In general, there is an observed trade-off. A sample eitherabsorbsa large amount of impact energy during the collision, even outperforming 'Foam' on occasionas with sample $C$ in Figure 7 , but at the cost of poor durability - wherein performance deteriorates rapidly after the first couple of hits. On the other end of the spectrum, the softer spine samples exhibit far greater durability, with nearly consistent absorption throughout all collisions, yet energy absorption is roughly equal, if not lower than 'Foam.'An ideal impact protection material balances these two parameters to match the needs of a given application. Perhaps further optimization of the impact technology could concurrently increase performance of both metrics so that one need not be sacrificed to realize the other.

\section{Conclusions}

This study is the first to investigatethe impact energy absorption capability of hedgehog spinesbeyond anecdotal biological evidence, measuring absorption trends over multiple impacts in a lab setting. Past studies have just suggested the possibility of applying the structural model to impact protection applications rather than demonstrating them. Additionally, no other study has investigated the effect of the densely packed spine arrangement analogous to the hedgehog model, nor have any parametric studies examined the effect of humidity conditioning on hedgehog spines. Our Results show that humidity softens keratin, making spines more durable but less energy absorbent. Greater impact speed decreases 
durability, but not initial energy absorption. It is also revealed that softer, elastic substrate adds energy absorption but samples deform more rapidly.

When samples are arranged in an orientation analogous to the natural model, hedgehog spines demonstrate impact absorption capabilities that confirm their role in the protection of hedgehogs during falls. This study demonstrates that in certain conditions, hedgehog spines can absorb as much, if not more, than industry standard impact-absorbing foam. However, there is a definite balance between impact absorption and multi-hit durability, as samples that absorb greater amount of impact energy remain intact for fewer hits due to greater damage, while other samples that absorb less impact energy have greater resilience and canendure significantly more collisions. However, these results only confirm the capabilities of the spine system as a whole. Further studies are still neededto understand the specific roles in impact absorption and durability played by the spines' internal structure, the layout of multiple adjacent spines, the material of the spines, the specific contribution by the substrate material, and a methodology to better control the 'flattening' of spines against the substrate, as each of these variables could alter results.

This investigation is one of the first of likely many steps towards designing and optimizing higher performing engineered impact and structural materials. By demonstrating how hedgehog spines exhibit similar impact energy absorption as a sample of foam from a Riddell Speed Classic football helmet, the authors provide evidence that hedgehog spines are a viable biomimetic model for a commercial impact resistantmaterial. Afterall, the spines used in this study were harvested from a dead animal, and despite the researchers' best efforts to maintain them, they may have deteriorated prior to testing.Specially designed and synthetically engineeredspine structures-built using3D additive manufacturing or other means-could be optimized for even better impact protection, as demonstrated with the microstructure found in Chiton teeth (de Obaldia et al. 2015).Biomimicry has proven time and again to be a reliable and fruitful innovation technique for inspiring new products such as VELCRO, which was developed based on how burrs stick to animal fur (Dickinson 1999).Perhaps a hedgehog-inspired impact protection technology isa forthcoming feat of biomimicry.

\section{Acknowledgements}

The authors would like to express their sincere thanks to Dr. Wieslaw Binienda (The University of Akron, UA) for use of facilities and testing equipment, to Dr. Todd Blackledge (UA) for providing ProAnalyst M otion Analysis Software, to Daphne Fecheyr-Lippens (UA) for taking SEM images of the spines, to Dr. Boyce Collins (North Carolina A\&T State University) for taking nano CT images of the spines, to John Swift and Preston Foster for preparing and donating the hedgehog pelt from which spines samples were used, toDoug Paige for experimental assistance, and to Samuel Kimble for his advice during the paper writing process. The authors also acknowledge the National Science Foundation for funding the experiment through the UA I- 
Corps Sites Grant (Award number 1000001693NSF). KT Tan would also like to acknowledge the start-up grant support provided by UA.

\section{References}

Ashby, M ichael F. 2005. M aterials Selection in M echanical Design. Burlington, M A: Elsevier ButterworthHeinemann.

Beukers, Adriaan, and Ed van Hinte. 2005. Lightness: the inevitable renaissance of minimum energy structures. 4th rev. Rotterdam: 010 Publishers.

Brazier, L. G. 1927. On the Flexure of Thin Cylindrical Shells and other "Thin" Sections. Proceedings of the Royal Society of London. Series A, Containing Papers of a Mathematical and Physical Character (The Royal Society) 116 (773): 104-114.

Bruet, B.J.F., Song, J., Boyce, M.C, Ortiz, C., 2008. M aterials design principles of ancient fish armor. Nat. M ater. 7 , 748-758.

Carlier, E. W. 1893. Contributions to the Histology of the Hedgehog (Erinaceus Europeus). Journal of Anatomy and Physiology. 27 (pt. 3): 169-178.

Calladine, C. R. 1983. The Brazier effect in the buckling of bent tubes. In Theory of Shell Structures., 595-625. Cambridge: Cambridge University Press.

Chen, I.H., Kiang, J.H., Correa, V., Lopez, M .I., Chen, P.Y., M cKittrick, J. and M eyers, M.A., 2011. Armadillo armor: mechanical testing and micro-structural evaluation. J. Mech. Behav. Biomed. Mater. 4, 713-722.

Chintapalli, R.K., M irkhalaf, M., Dastjerdi, A.K. and Barthelat, F., 2014. Fabrication, testing and modeling of a new flexible armor inspired from natural fish scales and osteoderms. Bioinspir. Biomim. 9, 036005.

Cho, Woo Kyung, James A. Ankrum, Dagang Guo, Shawn A. Chester, Seung Yun Yang, Anurag Kashyap, Georgina A. Campbell, et al. 2012. M icrostructured barbs on the North American porcupine quill enable easy tissue penetration and difficult removal.Proceedings of the National Academy of Sciences of the United States of America 109 (52): 21289-21294.

Chou, S. F., and R. A. Overfelt. 2011. Tensile deformation and failure of North American porcupine quills.M aterials Science and Engineering C 31: 1729-1736.

Chou, S. F., R. A. Overfelt, and M. E. Miller. 2012. Anisotropic mechanical behavior of keratin tissue from quill shells of North American porcupine (Erethizon dorsatum).M aterials Science \& Engineering A 557: 36-44.

Curiskis, J. I., and M. Feughelman. 1983. Finite Element Analysis of the Composite Fiber, Alpha Keratin. Textile Research Journal 271-274.

de Obaldia, Enrique Escobar, Chanhue Jeong, Lessa Kay Grunenfelder, David Kisailus, and Pablo Zavattieri. 2015. Analysis of the mechanical response of biomimetic materials with highly oriented microstructures through 3D printing, mechanical testing and modeling.Journal of the M echanical Behavior of Biomedical Materials 48: 70-85.

Dickinson, M ichael H. 1999. Bionics: Biological Insight into Mechanical Design.Proceedings of the National Academy of Sciences 96 (25): 14208-9. doi:10.1073/pnas.96.25.14208.

Gibson, Lorna J., Michael F. Ashby, and Brendan A. Harley. 2010. Cellular Materials in Nature and M edicine. New York: Cambridge University Press.

Guskiewicz, Kevin M., and Jason P. Mihalik. 2011. Biomechanics of Sport Concussion: Quest for the Elusive Injury Threshold.Exercise and Sport Sciences Reviews 4-11. http://www.medscape.com/viewarticle/735057_1.

Huang, J., Durden, H. and Chowdhury, M., 2011. Bio-inspired armor protective material systems for ballistic shock mitigation. Mater. Design 32, 3702-3710.

Karam, G. N., and L. J. Gibson. 1994. Biomimicking of animal quills and plant stems: natural cylindrical shells with foam cores.M aterials Science and Engineering C2: 113-132.

Matthews, L. Harrison. 1963. British Mammals. Collins.

Matthews, L. Harrison. 1974. Life of Mammals: v. 1 (Natural History). Littlehampton Book Services Ltd. 
McKittrick, J., P. Y. Chen, S. G. Bodde, W. Yang, E. E. Novitskaya, and M. A. M eyers. 2012. The Structure, Functions, and Mechanical Properties of Keratin.Journal of Materials 64 (4): 449-468.

M ohazzabi, Pirooz. 2011. When Does Air Resistance Become Significant in Free Fall?The Physics Teacher (American Association of Physics Teachers) 49 (2): 89.

Ommaya, A. K., and T. A. Gennarelli. 1974. Cerebral concussion and traumatic unconsciousness. Correlation of experimental and clinical observations of blunt head injuries.Brain 97 (4): 633-54.

Pellman, E. J., J. W. Powell, D. C. Viano, I. R. Casson, A. M. Tucker, H. Feuer, M. Lovell, J. F. Waeckerle, and D. W. Robertson. 2004. Concussion in professional football: epidemiological features of game injuries and review of the literature--part 3.Neurosurgery 54 (1): 81-96.

Reeve, Nigel. 1994. Hedgehogs. T \& A. D Poyser.

Rudykh, S., Ortiz, C. and Boyce, M .C., 2015. Flexibility and protection by design: imbricated hybrid microstructures of bio-inspired armor. Soft M atter 11, 2547.

Song, J., Ortiz, C. and Boyce, M.C., 2011. Threat-protection mechanics of an armored fish. J. M ech. Behav. Biomed. Mater. 4, 699-712.

Torres, G. Fernando, Omar O. Troncoso, John Diaz, and Diego Arce. 2014. Failure analysis of porcupine quills under axial compression reveals their mechanical response during buckling.Journal of the Mechanical Behavior of Biomedical Materials 39: 111-118.

Ucar, M., and A. Cengiz. 2012. Crash Pendulum" Energy Absorption Test System. Experimental Techniques (Society for Experimental Mechanics) 36: 33-38.

Vincent, J. F. V., and P. Owers. 1986. M echanical Design of Hedgehog Spines and Porcupine Quills.Journal of Zoology $55-75$.

Yang, W., C. Chao, and J. McKittrick. 2013. Axial compression of a hollow cylinder filled with foam: A study of porcupine quills.Acta Biomaterialia. 9: 5297-5304. 
(a)

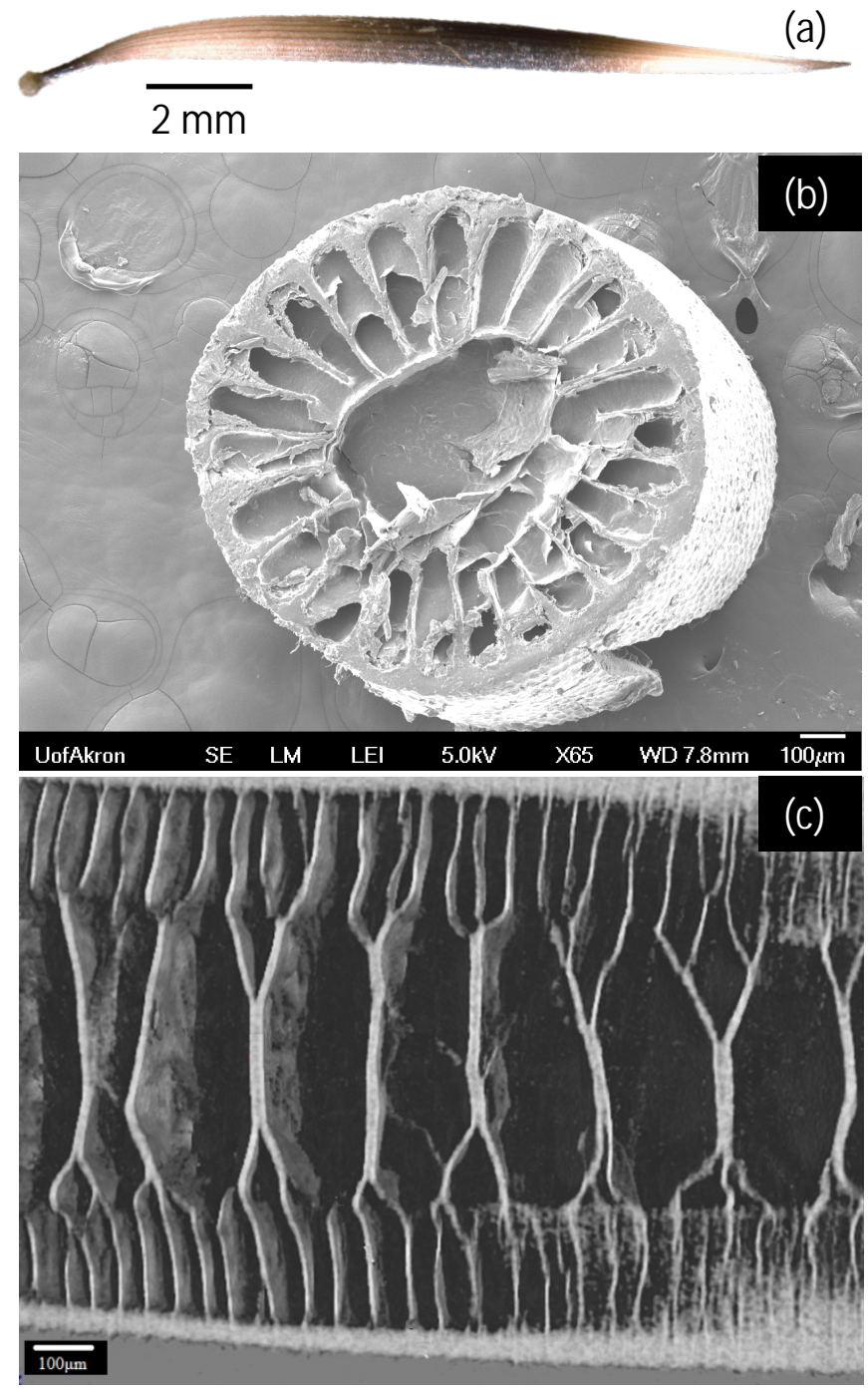

Figure 1: (a) Photograph of a hedgehog spine, showing the bulbed end on the left, which attaches to the animal; (b) SEM of a spine's lateral cross-section; (c) CT scan of a spine's longitudinal cross-section.

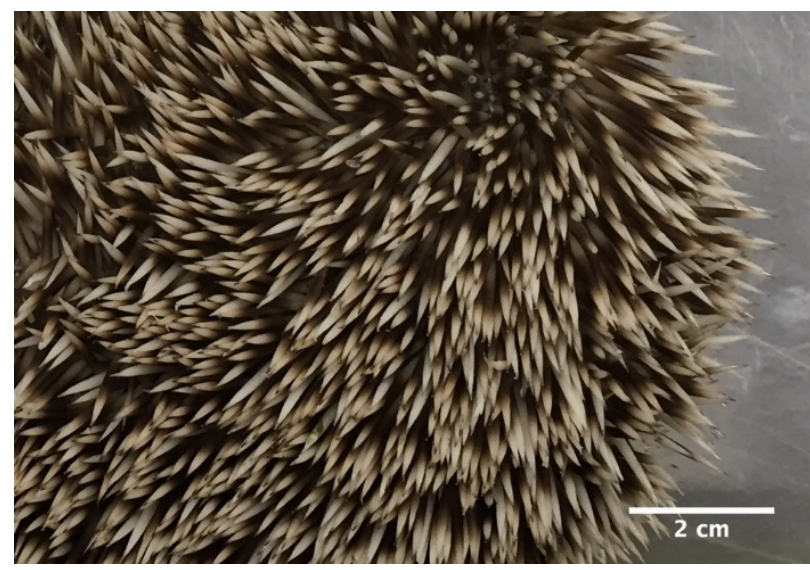

Figure 2: Densely packed spines embedded in the pelt of a dead hedgehog. 

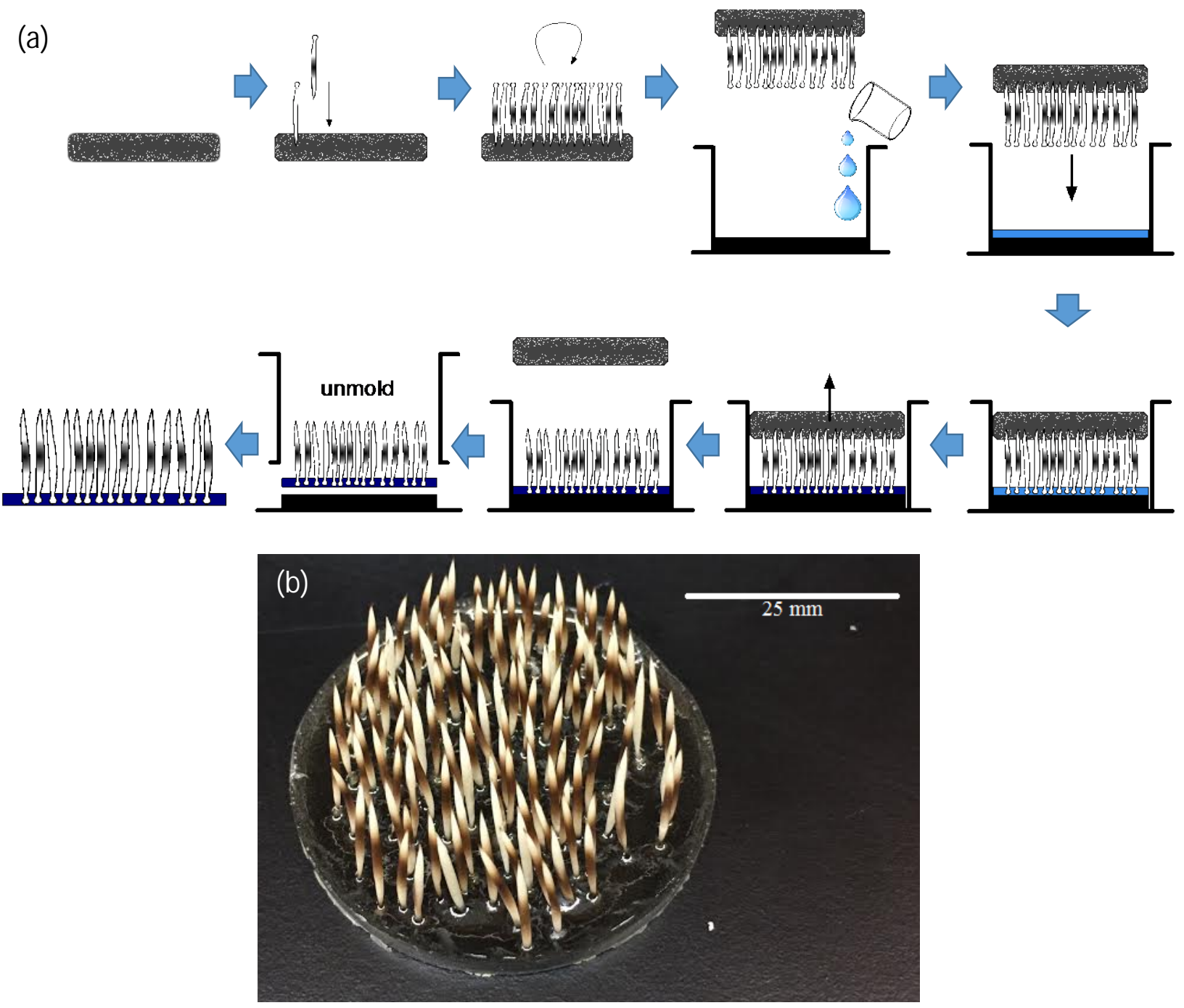

Figure 3: (a) Schematic of sample preparation; (b) A sample of hedgehog spines mounted vertically in a polyurethane (PU) substrate prior to testing. 

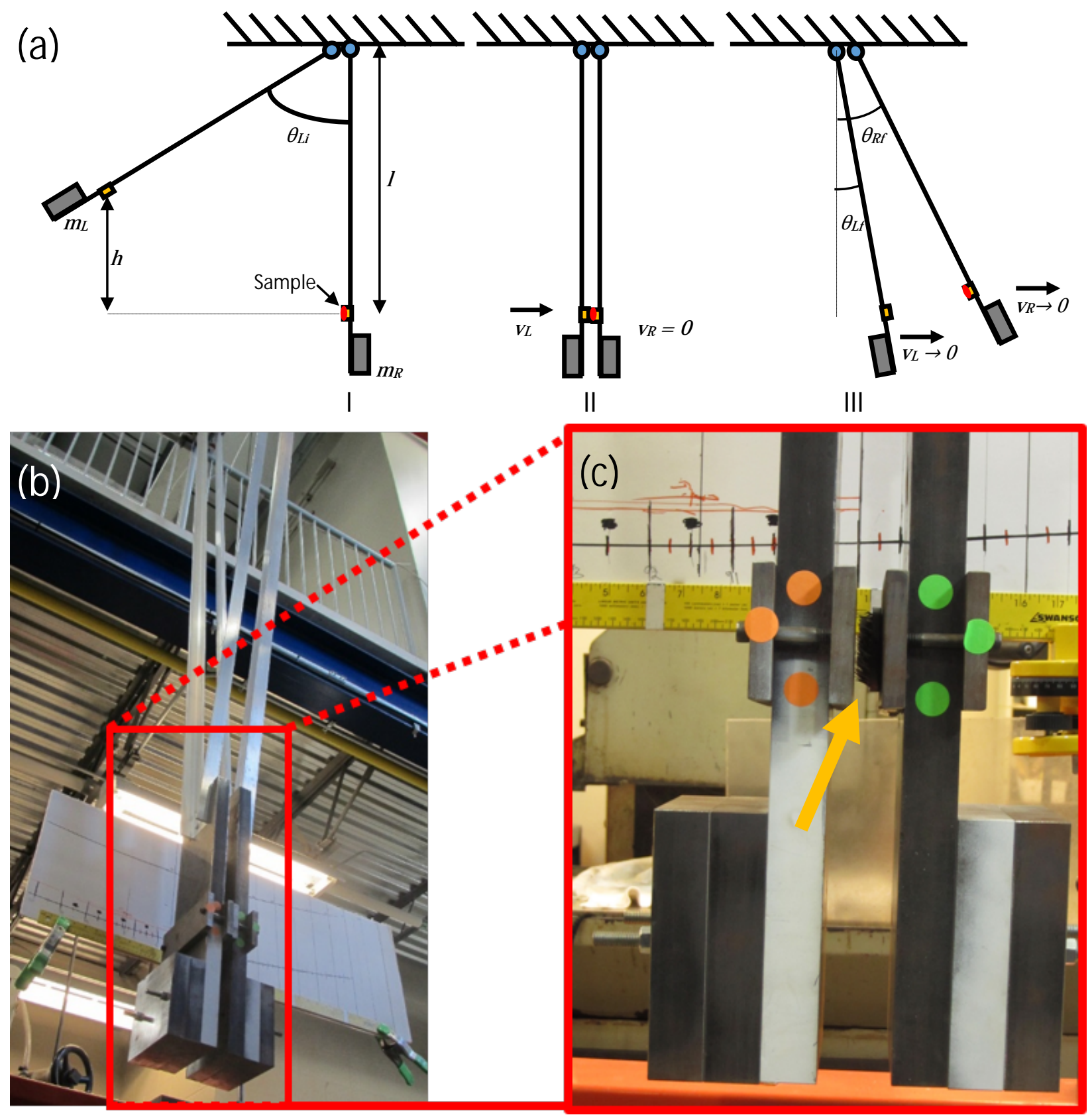

Figure 4: (a) Schematic of the pendulum apparatus arm motion during the course of one collision trial, with initial starting position (I), moment of collision (II), and post-collision back-swing (III); (b) Photo of the apparatus at rest; (c) Zoom-in detailing the colored stickers for video tracking and the location of the mounted sample (yellow arrow). 
(a)

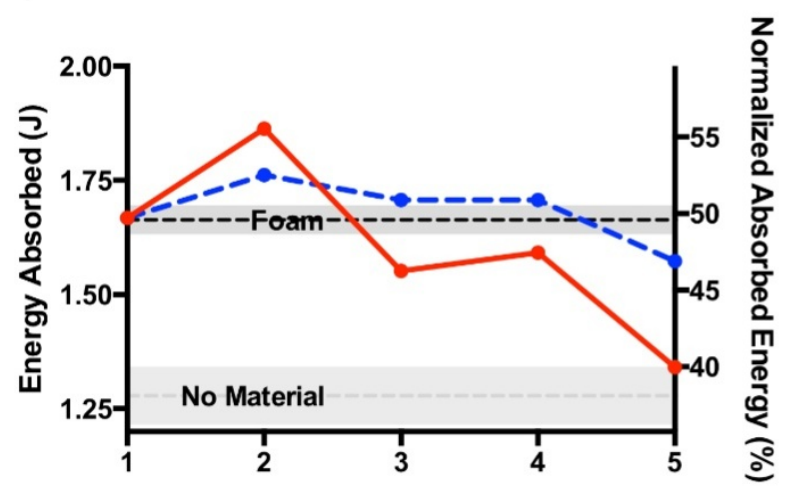

(b)

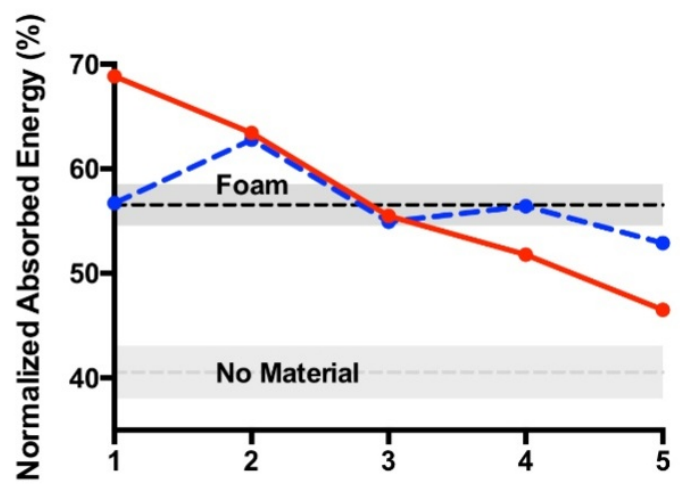

\section{$\longrightarrow[$ [ ] Dry Spines $\quad \longrightarrow-[$ [B] Humid Spines}

Figure 5:Energy absorbed over repeated low energy (3J) collisions. (a) Energy absorption calculated using the potential energy difference experimental technique. (b) Energy absorption calculated using the kinetic energy difference experimental technique (see supplementary). 'Foam' and 'No M aterial' are plotted as mean \pm s.d. Symbol (•) indicates low energy collisions. Blue is humid, red is dry.

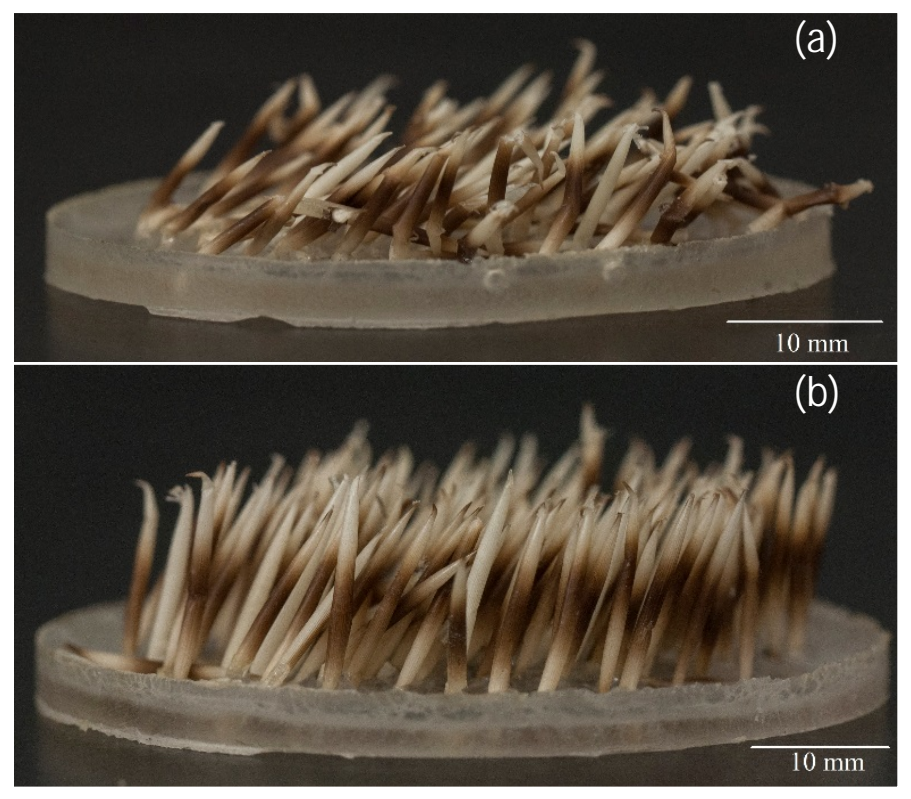

Figure 6: (a) Spines in Sample [A] show substantial breakage after five low-energy collisions; (b) In contrast, the spines in Sample [B] remain mostly intact after five low-energy collisions and five additional high-energy collisions. 
(a)

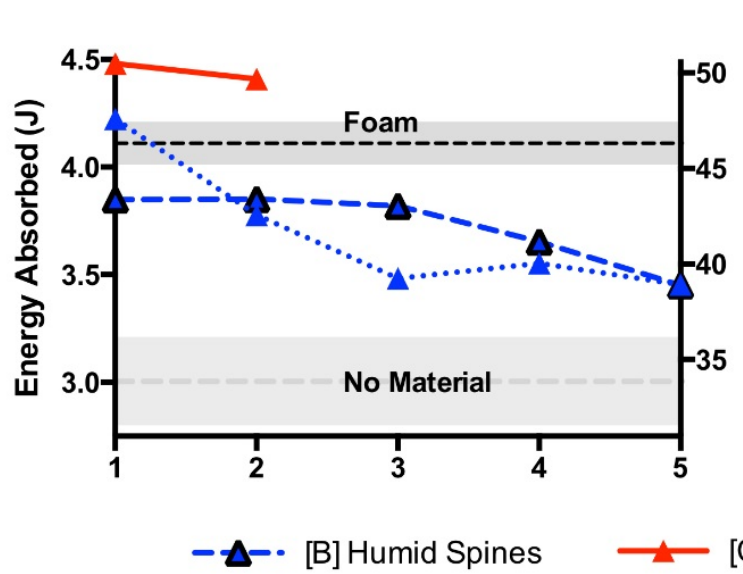

(b)
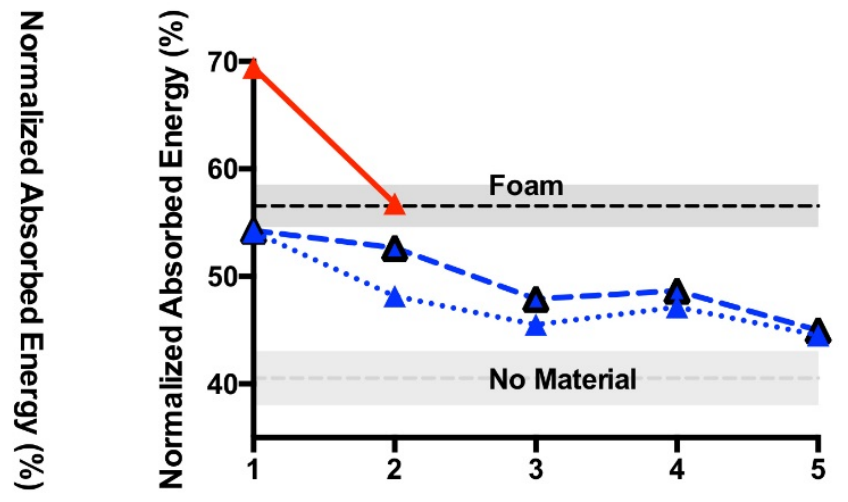

[C] Dry Spines $\cdots \wedge$ [... [D] Humid Spines

Figure 7: Energy absorbed over repeated high energy (8)) collisions. (a) Energy absorption calculated using the potential energy difference experimental technique. (b) Energy absorption calculated using the kinetic energy difference experimental technique (see supplementary). 'Foam' and 'No M aterial' are plotted as mean \pm s.d. Symbol $(\boldsymbol{\Delta})$ indicates high-energy collisions. Blue is humid, red is dry.
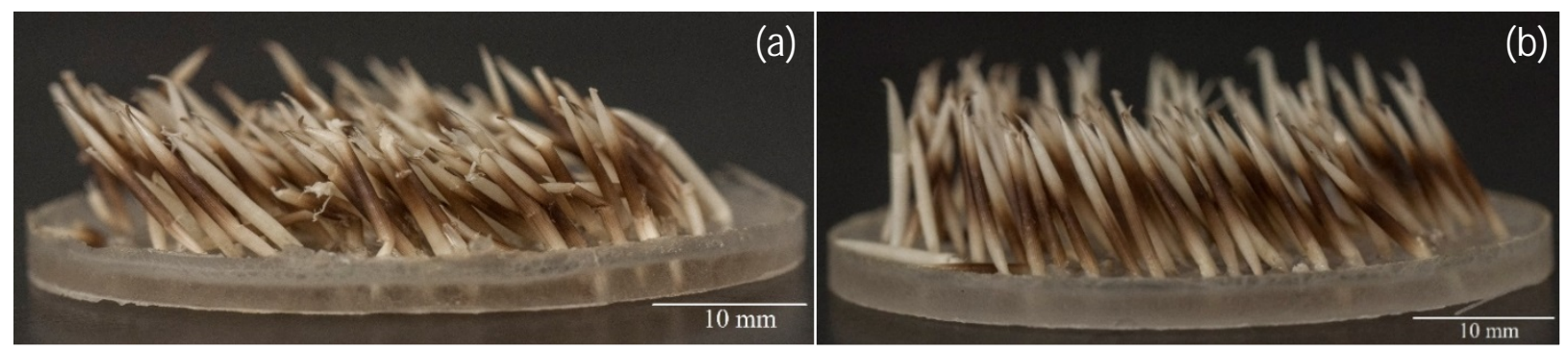

Figure 8: (a) Spines of dry Sample [C] show significant breakage after just two 8 Joule collisions; (b) M ost spines of humid Sample [D] remain intact with just minor damage after five consecutive 8 Joule collisions. 
(a)

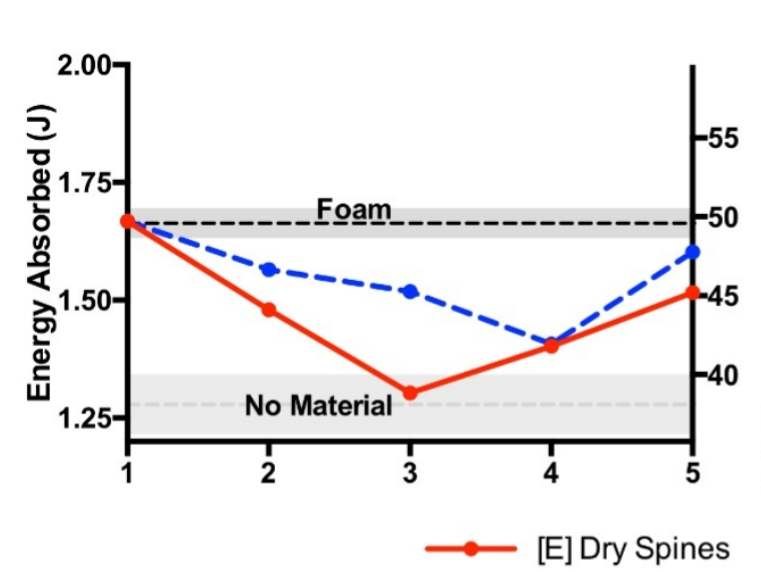

(c)

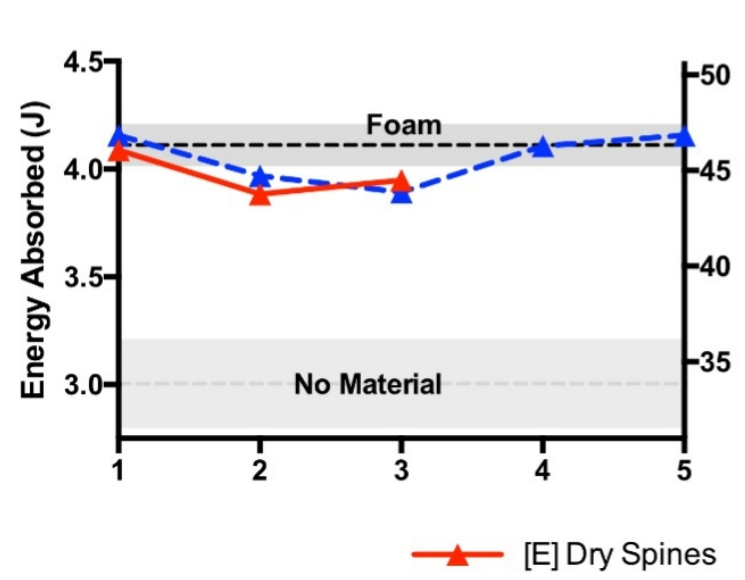

(b)

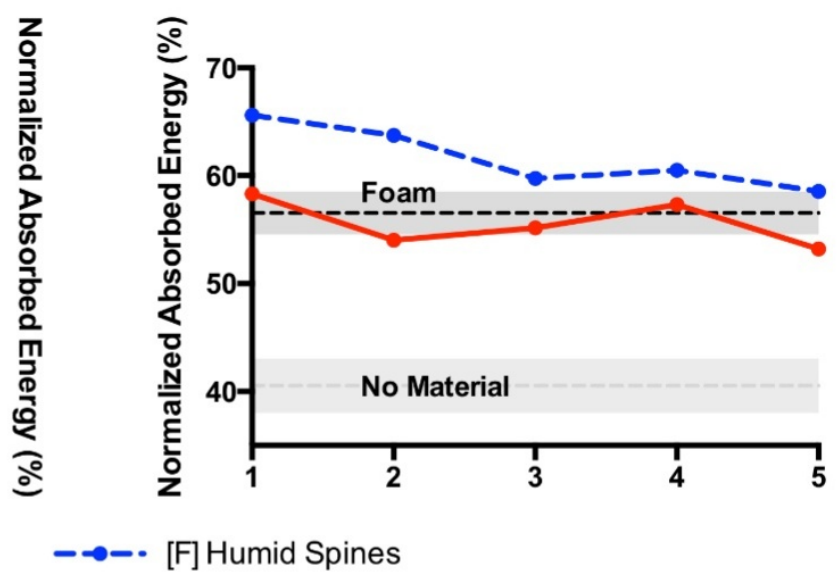

(d)

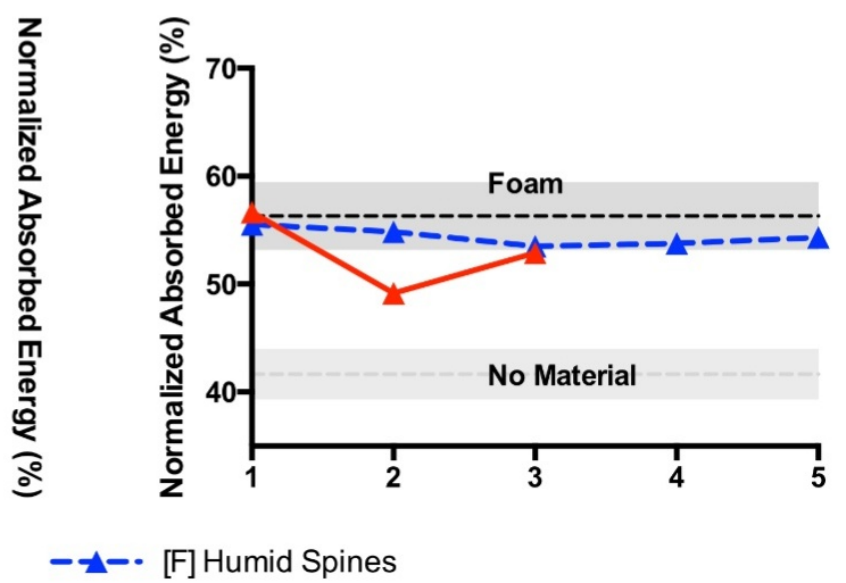

Figure 9: Energy absorbed by samples embedded in polyurethane (PU) substrates over repeated collisions. $(a, c)$ Energy absorption calculated using the potential energy difference experimental technique). (b,d) Energy absorption calculated using the kinetic energy difference experimental technique

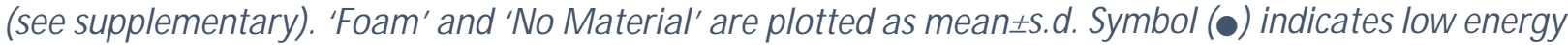
collisions; ( $\mathbf{\Lambda})$ indicates high-energy collisions. Blue is humid, red is dry. 

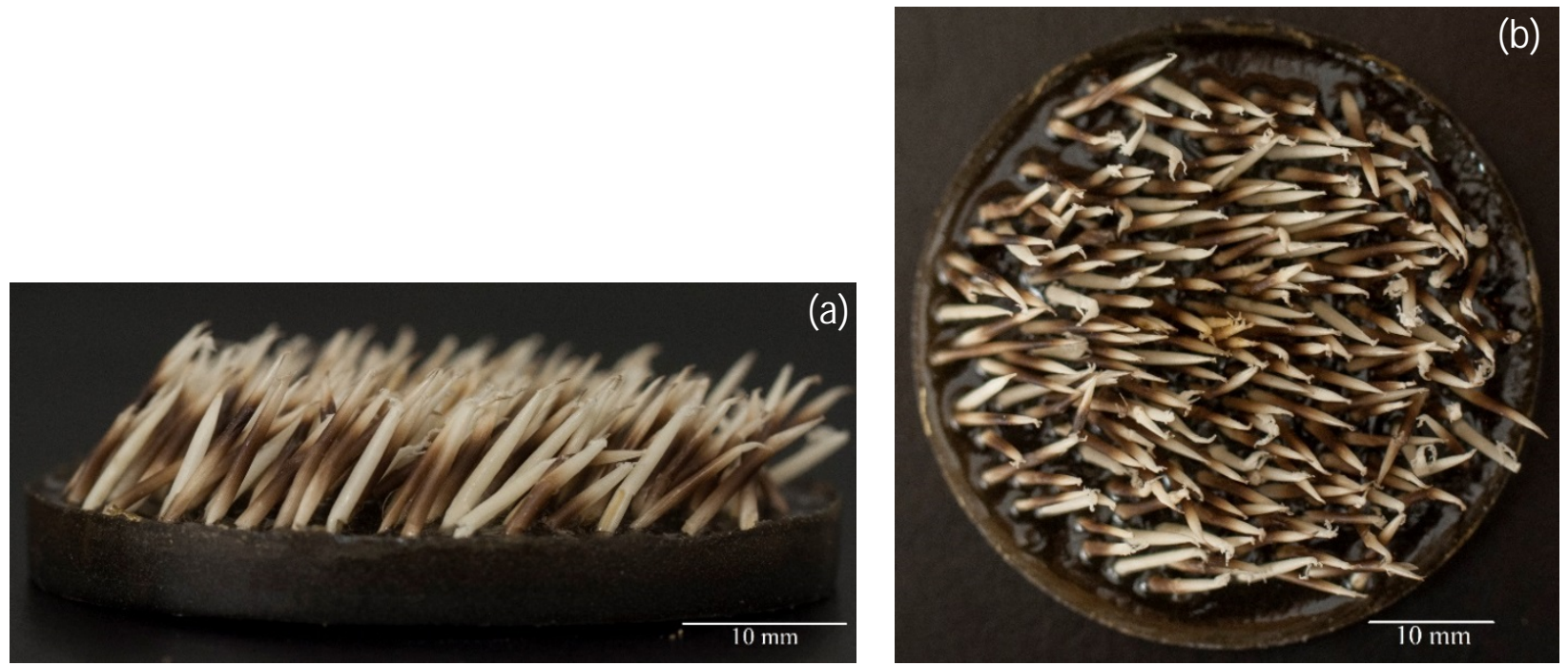

Figure 10: (a) Side and (b) Top view of Sample [E]. Although some spines broke, many were pressed flat against the PU substrate.

(a)

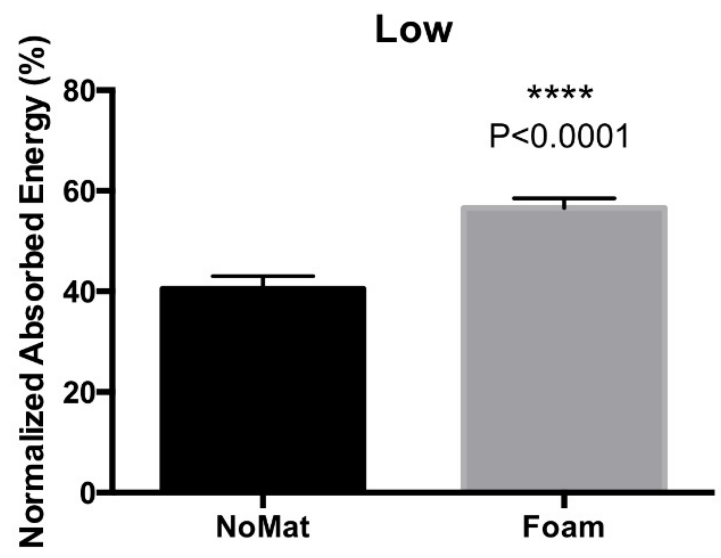

(b)

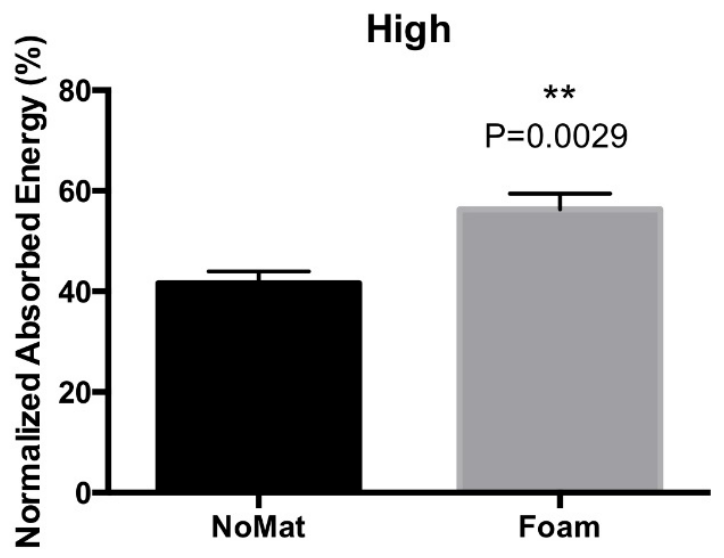

Figure 11: Column plot (mean \pm s.d.) showing significantly different impact absorption ability of 'Foam' and 'No M aterial' across all collisions. (a) Energy absorption in low energy tests; (b) Energy absorption in high-energy tests. In both low and high-energy tests, 'Foam' always shows significantly higher energy absorption ability over 'No M aterial.' ** indicates $\mathrm{P} \varangle 0.01$, **** indicates $\mathrm{P} \varangle 0.001$. 
(a)

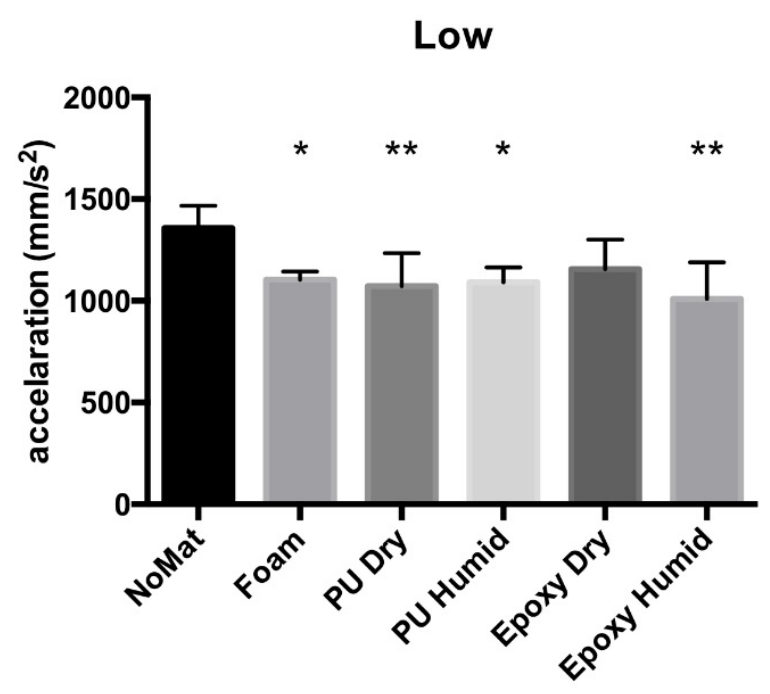

(b)

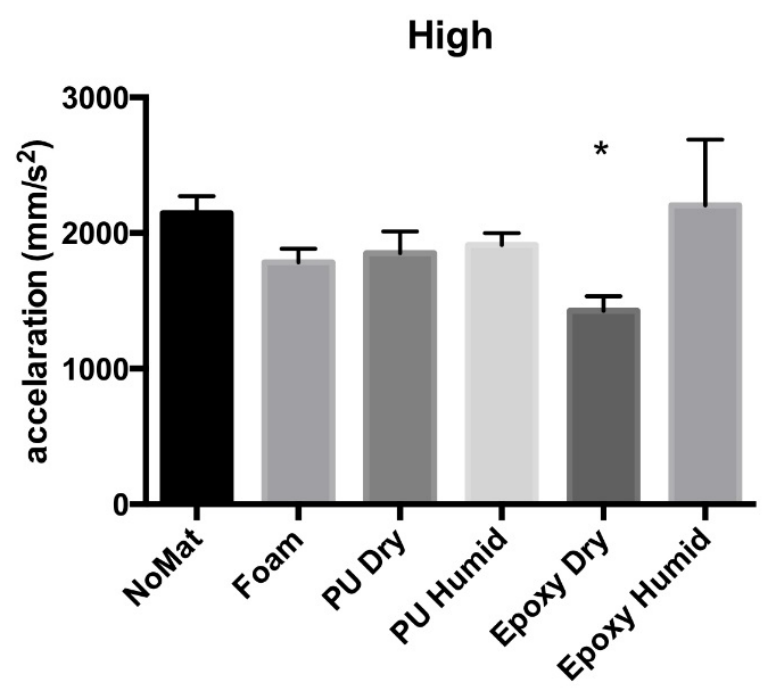

Figure 12: Column plot (mean \pm s.d.) showing maximum acceleration experienced by the impacted (right) pendulum across all collisions. (a) Compared to 'No M aterial,' all samples except Epoxy Dry (sample A) experienced significantly lower acceleration in low energy tests. (b) Compared to 'No Material,' only sample A (Epoxy Dry) experienced significantly low acceleration in high-energy tests. * indicates $\mathrm{P} \varangle 0.05$, ** indicates $\mathrm{P} \varangle 0.01$. 
Table 1: List of test samples, their relevant characteristics, and total collisions endured.

\begin{tabular}{cccc} 
Sample & Substrate & Humidity & Total Collisions (Impact Energy) \\
\hline A & Epoxy & Dry & 5 (Low) \\
B & Epoxy & Humid & 5 (Low); 5 (High) \\
C & Epoxy & Dry & 2 (High) \\
D & Epoxy & Humid & 5 (High) \\
E & PU & Dry & $5($ Low); 3 (High) \\
F & PU & Humid & $5($ Low); 5 (High) \\
\hline
\end{tabular}

